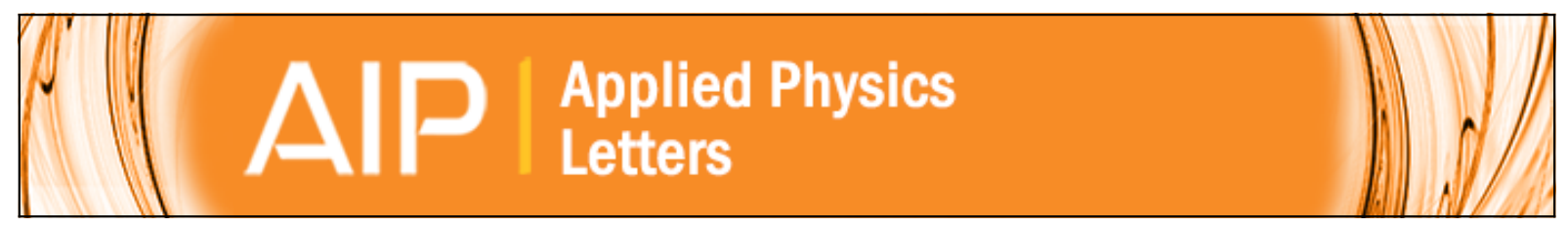

\title{
Giant magnetothermopower associated with large magnetoresistance in $\mathrm{Ag} 2 \mathrm{Te}$
}

Young Sun, M. B. Salamon, M. Lee, and T. F. Rosenbaum

Citation: Applied Physics Letters 82, 1440 (2003); doi: 10.1063/1.1558896

View online: http://dx.doi.org/10.1063/1.1558896

View Table of Contents: http://scitation.aip.org/content/aip/journal/apl/82/9?ver=pdfcov

Published by the AIP Publishing

\section{Articles you may be interested in}

A three-dimensional resistor network model for the linear magnetoresistance of Ag $2+$ Se and $\mathrm{Ag} 2+\mathrm{Te}$ bulks J. Appl. Phys. 104, 113922 (2008); 10.1063/1.3035834

Low temperature thermal, thermoelectric, and thermomagnetic transport in indium rich $\mathrm{Pb} 1 \times \mathrm{Sn} \times \mathrm{Te}$ alloys J. Appl. Phys. 103, 053710 (2008); 10.1063/1.2890150

Transport properties of metallic La $1 \times \mathrm{Sr} \times \mathrm{CoO} 3(0.30 \times 0.50)$ ferromagnet

J. Appl. Phys. 100, 103912 (2006); 10.1063/1.2363236

Magnetoresistance in $\mathrm{Ag} 2+$ Se with high silver excess Appl. Phys. Lett. 86, 072102 (2005); 10.1063/1.1866642

Thermoelectric properties of Ag 3 AuTe 2

J. Appl. Phys. 88, 5221 (2000); 10.1063/1.1316057

\section{A|P| $\left.\right|_{\text {Applied Physics }} ^{\text {Journal of }}$}

Journal of Applied Physics is pleased to announce André Anders as its new Editor-in-Chief 


\title{
Giant magnetothermopower associated with large magnetoresistance in $\mathrm{Ag}_{2-\delta} \mathrm{Te}$
}

\author{
Young Sun ${ }^{\mathrm{a})}$ and M. B. Salamon \\ Department of Physics and Materials Research Laboratory, University of Illinois at Urbana-Champaign, \\ Urbana, Illinois 61801 \\ M. Lee and T. F. Rosenbaum \\ The James Franck Institute and Department of Physics, The University of Chicago, Chicago, Illinois 60637
}

(Received 2 December 2002; accepted 13 January 2003)

\begin{abstract}
We have probed the temperature and magnetic-field dependence of the thermopower and resistance of a $p$-type silver chalcogenide, $\mathrm{Ag}_{2-\delta} \mathrm{Te}$. The application of a magnetic field causes not only a large magnetoresistance but also a giant magnetothermopower effect. The maximum change of thermopower is as high as $470 \mu \mathrm{V} / \mathrm{K}$ in a $7 \mathrm{~T}$ magnetic field. Both the magnetoresistance and the magnetothermopower show a pronounced peak and nearly linear behavior near the sign change of the thermopower. Bandcrossing and quantum confinement due to disorder appear to play key roles in the heightened response to field. (C) 2003 American Institute of Physics.
\end{abstract}

[DOI: $10.1063 / 1.1558896]$

Application of a magnetic field often causes large changes of physical properties of condensed matter. The prototype examples are the so-called "giant magnetoresistance" (GMR) and "colossal magnetoresistance" (CMR) effects, i.e., huge changes of resistance under magnetic field, in magnetic materials as diverse as magnetic multilayers, ${ }^{1}$ magnetic granular systems, ${ }^{2}$ and perovskite manganites. ${ }^{3}$ In 1997, Xu et al. reported a large magnetoresistance (MR) effect in silver chalcogenides, $\mathrm{Ag}_{2 \pm \delta} \mathrm{Se}$ and $\mathrm{Ag}_{2 \pm \delta} \mathrm{Te}$, which extended the GMR/CMR family to nonmagnetic compounds. ${ }^{4}$ Since then, the silver chalcogenies have received much attention. $^{5-7}$

The low temperature $(\alpha)$ phase of the silver chalcogenides is a narrow band gap semiconductor. At high temperatures $(\sim 400 \mathrm{~K})$ these compounds undergo a phase transition into a superionic conducting state. Perfectly stoichiometric $\mathrm{Ag}_{2} \mathrm{Se}$ and $\mathrm{Ag}_{2} \mathrm{Te}$ have negligible $\mathrm{MR},{ }^{8}$ but small amounts of excess silver or excess Se/Te lead to large MR effects comparable to the CMR in perovskite manganites. Compared with those GMR/CMR materials, silver chalcogenides have several distinct features: (i) they are nonmagnetic compounds while both GMR multilayers/granular systems and CMR manganites are magnetic materials; (ii) the MR is positive while the usual GMR/CMR are negative; (iii) in some cases the MR shows a linear dependence on applied magnetic field; ${ }^{4}$ and (iv) the MR does not saturate even in a magnetic field as high as $60 \mathrm{~T}^{7}$ These features imply that some unconventional physics underlies the MR in silver chalcogenides. In particular, Abrikosov has proposed a theory named "quantum magnetoresistance" to account for the unusual linearity of MR over a large field range. ${ }^{9,10}$ An essential element in this theory is a semiconducting gap that approaches zero when two bands cross, with a linear energy spectrum. The model depends as well on the proclivity of the excess/deficient silver to form an inhomogeneous medium in

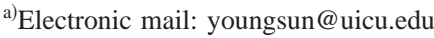

which metallic clusters are embedded in a less conducting matrix. This theory has been supported by recent experiments. By tuning the band structure of $\mathrm{Ag}_{2-} \mathrm{Te}$ using hydrostatic pressure, Lee et al. found that the MR peaks and becomes linear in field just when the electron and hole bands cross. In this letter, we report another giant effect produced by applied magnetic field, i.e., a giant magnetothermopower (GMTEP), in a $p$-type $\mathrm{Ag}_{2-\delta} \mathrm{Te}$ sample. The maximum change of thermopower (TEP) is as high as $470 \mu \mathrm{V} / \mathrm{K}$ in a 7 $\mathrm{T}$ field. Moreover, both GMTEP and MR show a pronounced peak and an almost linear behavior near the sign change of TEP, suggesting that the bandcrossing and quantum confinement due to disorder are key elements of the physics.

Appropriately weighted amounts of high purity $\mathrm{Ag}$ (99.999\% pure, Alfa Aesar) and Te (99.999\% pure, metals basis, Alfa Aesar), sealed in quartz tubes under a vacuum better than $5 \mathrm{mTorr}$, were melted to create polycrystalline samples at desired stoichiometries. The compound was rocked at $50{ }^{\circ} \mathrm{C}$ above the reported melting point to ensure complete mixing. Slowly cooled samples were cut perpendicular to the long axis of the cylindrical boule to avoid dopant variations due to small temperature gradients in the furnace. In addition, the sample in this study was fashioned as a long narrow piece of typical dimensions $(1 \times 1 \times 8) \mathrm{mm}^{3}$ to circumvent the geometrical effects that have been associated with high carrier mobility semiconductors. Resistance and thermopower were measured using standard methods. The sample was attached to the cold finger of a cryostat with a resistive chip heater varnished at the hot end. Two differential type- $E$ thermocouples were varnished on the sample to measure the temperature gradient, and voltage leads (fine $\mathrm{Au}$ wires) were placed close to the thermocouples to measure the potential difference. Current leads (fine $\mathrm{Au}$ wires) were placed outside the region between the thermocouples, so that resistance and TEP data could be taken in sequence at each temperature-field point. The magnetic field was applied normal to the long axis of the sample. 


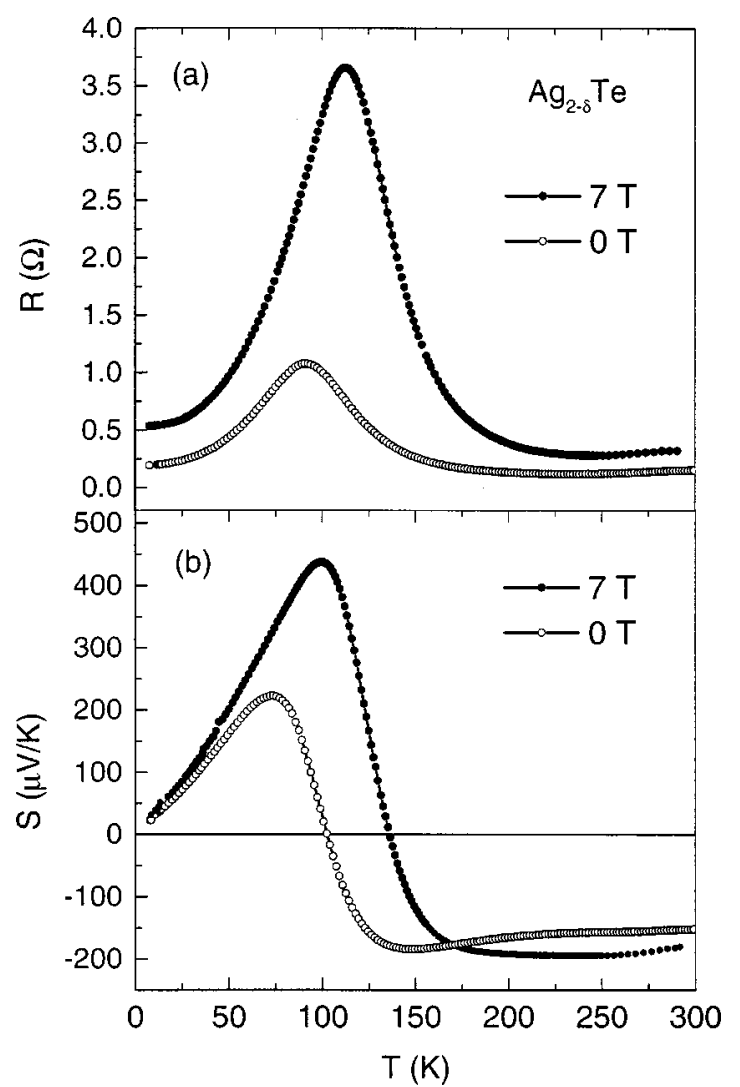

FIG. 1. Temperature dependence of (a) resistance and (b) thermopower in 0 and $7 \mathrm{~T}$ magnetic field for $\mathrm{Ag}_{2-\delta} \mathrm{Te}$.

We plot in Fig. 1(a) the temperature dependence of the resistance in 0 and $7 \mathrm{~T}$ field. The resistance data are consistent with previous reports. ${ }^{6}$ Below $100 \mathrm{~K}$, the resistance rises because the effective carrier density is constant and the phonon scattering increases with temperature. At high temperature, the transport becomes activated and resistance decreases with increasing temperature. The resistance peak around $100 \mathrm{~K}$ reflects a transition from the extrinsic to the intrinsic carrier regime of a narrow-gap semiconductor with increasing temperature. At $7 \mathrm{~T}$, the resistance peak is greatly enhanced and the metallic-semiconducting transition shifts to higher temperature, resulting in a large MR. The corresponding temperature dependence of $\Delta R=R(H)-R(0)$, is plotted in Fig. 2. The $\Delta R(T)$ exhibits a pronounced peak at $100 \mathrm{~K}$, which is a prominent feature of $p$-type silver chalcogenides. ${ }^{5}$

The temperature dependence of the thermopower, $S$, with and without magnetic field, is demonstrated in Fig. 1(b). At high temperature, $S$ is negative and decreases slightly with decreasing temperature. Below $140 \mathrm{~K}, S$ rises dramatically and a sign change from negative to positive occurs at $103 \mathrm{~K}$. Below $70 \mathrm{~K}, S$ shows a nearly linear dependence with decreasing temperature in accordance with the usual behavior of diffusion thermopower in metals. The $S(T)$ behavior suggests that the transport properties are dominated by electrons at high temperature and by holes at low temperature. The Hall coefficient of the same sample also changes sign around $100 \mathrm{~K},{ }^{11}$ consistent with the thermopower data. When under a $7 \mathrm{~T}$ field, $S$ is altered slightly at low temperature, but is greatly enhanced between 50 and $150 \mathrm{~K}$ so as to reverse the sign of $S$ and shift the zero point to $136 \mathrm{~K}$. Consequently, a GMTEP effect appears in this temperature range. The cor-

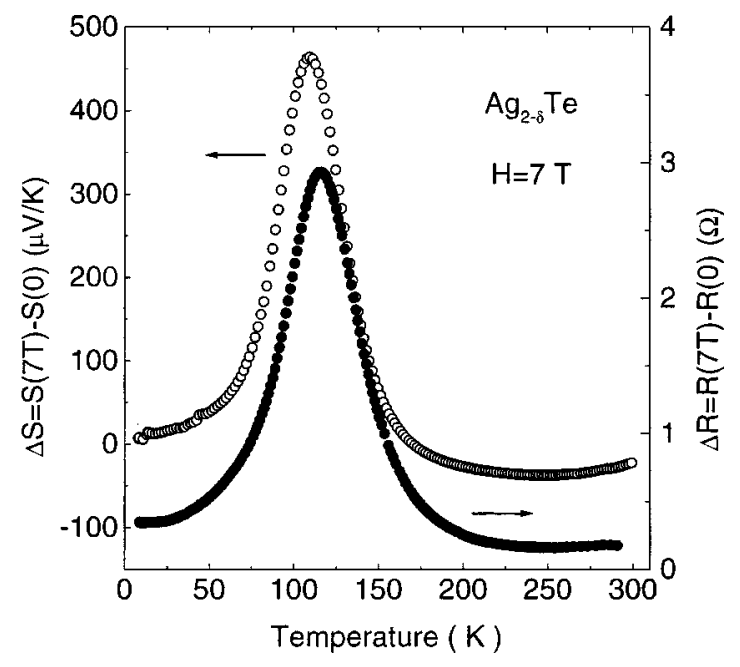

FIG. 2. Temperature dependence of magnetoresistance $(\Delta R)$ and magnetothermopower $(\Delta S)$ for $\mathrm{Ag}_{2-\delta} \mathrm{Te}$.

responding temperature dependence of the GMTEP is shown in Fig. 2. The maximum value of $\Delta S=S(H)-S(0)$ is about $470 \mu \mathrm{V} / \mathrm{K}$ at $110 \mathrm{~K}$. Such huge change of TEP under magnetic fields is rare and only reported in doped InSb. ${ }^{12,13} \mathrm{Al}-$ though the GMR multilayers and granular films, as well as the CMR manganites, have been reported to exhibit a socalled "giant magnethothermopower", ${ }^{14,15}$ the absolute values of $\Delta S$ for these materials are several to tens of $\mu \mathrm{V} / \mathrm{K}$, an order of magnitude smaller than that in $\mathrm{Ag}_{2}{ }_{\delta} \mathrm{Te}$.

From Fig. 2, it is clear that there is a close correlation between $\Delta R$ and $\Delta S$ : namely, they occur in the same temperature range and the peak temperature of $\Delta S$ is very close to that of $\Delta R$. The GMTEP is associated unequivocally with the large MR. Furthermore, the peaks of $\Delta R$ and $\Delta S$ lie around $100-110 \mathrm{~K}$, near the sign change of the thermopower. This characteristic highlights the pivotal role played by bandcrossing, in accordance with the study of band gap tuning by pressure in $p$-type silver chalcogenides, ${ }^{6}$ where the MR peaks just when the bands cross.

In order to draw more detailed information on how the resistance and thermopower vary with magnetic field, we also measured the field dependence of the MR and the magnetothermopower (MTEP) at various temperatures. The results are demonstrated in Figs. 3(a) and 3(b), respectively. The MR ratio, defined as $\mathrm{MR}=[R(H)-R(0)] / R(0)$, evolves from a superlinear field dependence at high temperatures $(150$ and $300 \mathrm{~K})$ to a sublinear field dependence at low temperatures $(8,70,100$, and $110 \mathrm{~K})$. A nearly linear field dependence emerges at $130 \mathrm{~K}$ where the MR ratio is maximum. Correspondingly, the MTEP is superlinear at high temperatures, sublinear at low temperatures, and nearly linear at $130 \mathrm{~K}$. These results further confirm the correlation between the MR and the MTEP in $\mathrm{Ag}_{2-\delta}$ Te. Nevertheless, it should be noted that a more complicated field dependence holds in MTEP. At both low $(8 \mathrm{~K})$ and high temperatures (300 and $150 \mathrm{~K}$ ), the MTEP exhibits a nonmonotonic behavior, which is absent in MR. Although this nonmonotonic behavior could suggest an orbital origin of MTEP, it is not well understood at this stage.

The close correlation between the MR and the GMTEP implies that they could share the same mechanism. Accord- 

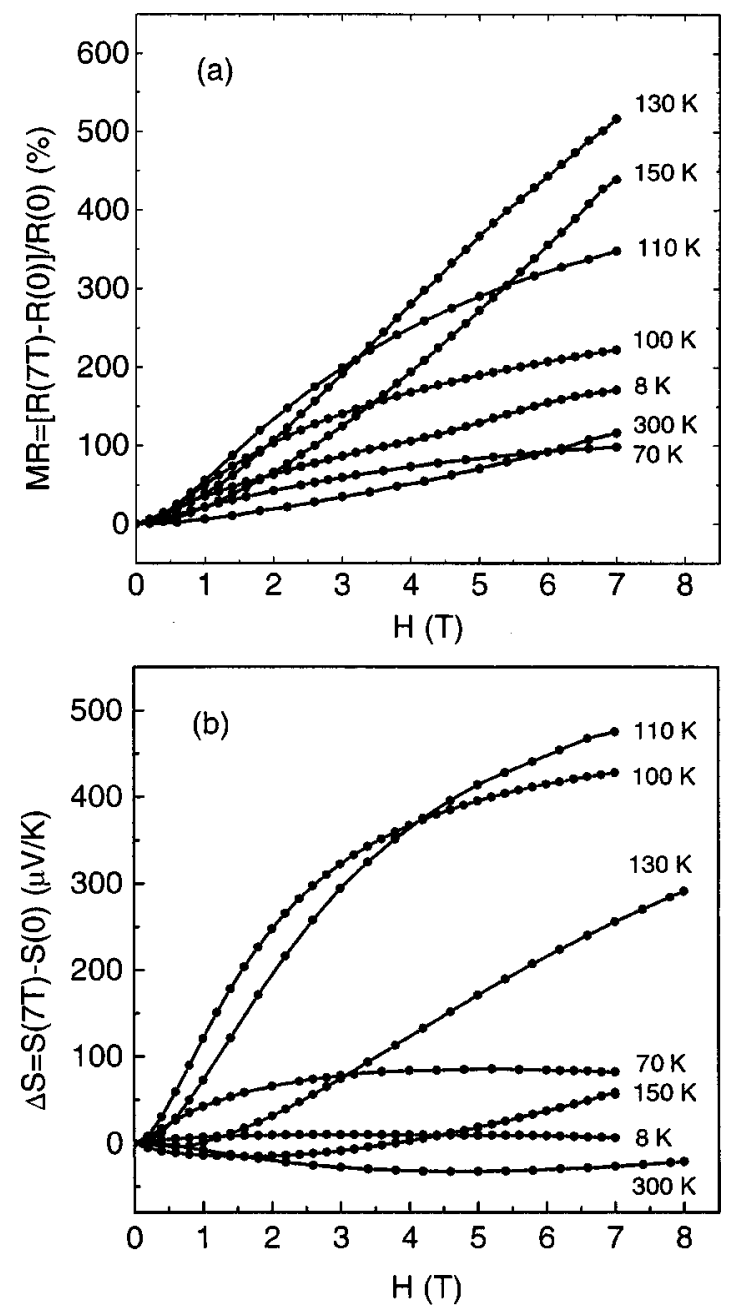

FIG. 3. Magnetic-field dependence of (a) MR ratio and (b) magnetothermopower $(\Delta S)$ at various temperatures for $\mathrm{Ag}_{2-\delta} \mathrm{Te}$.

ing to Abrikosov's theory, the electronic structure of the doped $\mathrm{Ag}_{2} \mathrm{Te}$ is close to that of a gapless semiconductor, which consists of crossed linear bands, with the chemical potential $\mu$ lying at the crossing point. At that point, there is electron-hole symmetry and zero thermoelectric power. The chemical potential depends on magnetic field and, through the doping level, on temperature. At high temperatures, the thermally excited electrons drive the system slightly $n$ type. The chemical potential then lies at higher energy than the crossing point. Lowering the temperature moves $\mu$ toward and through the crossing point, which leads to a sign change of the TEP. This occurs at $103 \mathrm{~K}$, where the zero-field chemical potential reaches the crossing point and the TEP is zero. Applying a magnetic field also lowers $\mu$ and moves it away from the crossing point into the hole-doped regime so that the $n$-type (negative) TEP in zero field becomes $p$-type (positive) in high fields and the GMTEP arises. This picture is consistent as well with the resistance data, where the MR is nearly linear just at the temperature where the in-field $\mu$ is at the crossing point.

Our understanding of the GMTEP must also consider the effects of disorder caused by nonstoichiometry. Abrikosov invokes an effective medium approach in his theory to account for the magnetoresistance over a large field range. Similarly, the excess/deficient silver regions (presumably in the form of small "wires" or nucleated "dots") could play a pivotal role in $\Delta S(T)$. Just as low dimensional systems with layers and chains in the microstructure show enhanced thermopower, ${ }^{16}$ the special form of quantum confinement in off-stoichiometric $\mathrm{Ag}_{2-\delta}$ Te could contribute to the GMTEP.

In summary, application of a magnetic field in $\mathrm{Ag}_{2}-{ }_{\delta} \mathrm{Te}$ causes not only a large magnetoresistance but also a giant magnetothermopower. Both the MR and the GMTEP show a pronounced peak and nearly linear behavior near the sign change of the TEP. We identify bandcrossing as well as quantum confinement due to disorder as key elements in the silver chalcogenides' extraordinary sensitivity to magnetic field.

The authors are grateful to Tuson Park for help in the thermopower measurements. The work at University of Illinois was partially supported by NSF Grant No. EIA0121568. The work at the University of Chicago was supported by US Department of Energy Grant No. DE-FG0299ER45789.

${ }^{1}$ M. N. Baibich, J. M. Broto, A. Fert, F. Nguyen van Dau, F. Petroff, P. Etienne, G. Creuzet, A. Friederich, and J. Chazeles, Phys. Rev. Lett. 61, 2472 (1998).

${ }^{2}$ A. E. Berkowitz, J. R. Mitchell, M. J. Carey, A. P. Young, S. Zhang, F. E. Spada, F. T. Parker, A. Hutten, and G. Thomas, Phys. Rev. Lett. 68, 3745 (1992).

${ }^{3}$ S. Jin, T. H. Tiefel, M. McCormack, R. A. Fastnacht, R. Ramesh, and L. H. Chen, Science 264, 413 (1994).

${ }^{4}$ R. Xu, A. Husmann, T. F. Rosenbaum, M.-L. Saboungi, J. E. Enderby, and P. B. Littlewood, Nature (London) 390, 57 (1997).

${ }^{5}$ H. S. Schnyders, M.-L. Saboungi, and T. F. Rosenbaum, Appl. Phys. Lett. 76, 1710 (2000)

${ }^{6}$ M. Lee, T. F. Rosenbaum, M.-L. Saboungi, and H. S. Schnyders, Phys. Rev. Lett. 88, 066602 (2002).

${ }^{7}$ A. Husmann, J. B. Betts, G. S. Boebinger, A. Migliori, T. F. Rosenbaum, and M.-L. Saboungi, Nature (London) 417, 421 (2002).

${ }^{8}$ P. Junod, Helv. Phys. Acta 32, 567 (1959).

${ }^{9}$ A. A. Abrikosov, Phys. Rev. B 58, 2788 (1998).

${ }^{10}$ A. A. Abrikosov, Europhys. Lett. 49, 789 (2000).

${ }^{11} \mathrm{M}$. Lee and T. F. Rosenbaum (unpublished).

${ }^{12}$ J. P. Heremans, C. M. Thrush, and D. T. Morelli, Phys. Rev. Lett. 86, 2098 (2001).

${ }^{13}$ J. P. Heremans, C. M. Thrush, and D. T. Morelli, Phys. Rev. B 65, 035209 (2001).

${ }^{14}$ J. Shi, K. Pettit, E. Kita, S. S. P. Parkin, R. Nakatani, and M. B. Salamon, Phys. Rev. B 54, 15273 (1996).

${ }^{15}$ M. Jaime, M. B. Salamon, K. Pettit, M. Rubinstein, R. E. Treece, J. S. Horwitz, and D. B. Chrisey, Appl. Phys. Lett. 68, 1576 (1996).

${ }^{16}$ M. S. Dresselhaus, Recent Trends in Thermoelectric Materials Research III, Semiconductors and Semimetals Vol. 71 (Academic, San Diego, 2001). 